\title{
Imaging the disk around IRAS 20126+4104 at subarcsecond resolution ${ }^{\star}$
}

\author{
R. Cesaroni ${ }^{1}$, D. Galli ${ }^{1}$, R. Neri ${ }^{2}$, and C. M. Walmsley ${ }^{1,3}$ \\ 1 INAF, Osservatorio Astrofisico di Arcetri, Largo E. Fermi 5, 50125 Firenze, Italy \\ e-mail: [cesa;galli;walmsley] @arcetri.astro.it \\ 2 Institut de Radioastronomie Millimétrique, 300 rue de la Piscine, 38406 Saint Martin d'Hères, France \\ e-mail: neri@iram.fr \\ ${ }^{3}$ Dublin Institute for Advanced Studies (DIAS), 31 Fitzwilliam Place, 2 Dublin, Ireland
}

Received 15 November 2013 / Accepted 9 May 2014

\section{ABSTRACT}

\begin{abstract}
Context. The existence of disks around high-mass stars has yet to be established on a solid ground, as only few reliable candidates are known to date. The disk rotating about the $\sim 10^{4} L_{\odot}$ protostar IRAS 20126+4104 is probably the most convincing of these.

Aims. We would like to resolve the disk structure in IRAS 20126+4104 and, if possible, investigate the relationship between the disk and the associated jet emitted along the rotation axis.

Methods. We performed observations at $1.4 \mathrm{~mm}$ with the IRAM Plateau de Bure interferometer attaining an angular resolution of $\sim 0$ '.4 ( $\sim 660 \mathrm{AU})$. We imaged the methyl cyanide $J=12 \rightarrow 11$ ground state and vibrationally excited transitions as well as the $\mathrm{CH}_{3}{ }^{13} \mathrm{CN}$ isotopologue, which had proved to be disk tracers.

Results. Our findings confirm the existence of a disk rotating about a 7-10 $M_{\odot}$ star in IRAS 20126+4104, with rotation velocity increasing at small radii. The dramatic improvement in sensitivity and spectral and angular resolution with respect to previous observations allows us to establish that higher excitation transitions are emitted closer to the protostar than the ground state lines, which demonstrates that the gas temperature is increasing towards the centre. We also find that the material is asymmetrically distributed in the disk and speculate on the possible origin of such a distribution. Finally, we demonstrate that the jet emitted along the disk axis is co-rotating with the disk.

Conclusions. We present iron-clad evidence of the existence of a disk undergoing rotation around a B-type protostar, with rotation velocity increasing towards the centre. We also demonstrate that the disk is not axially symmetric. These results prove that B-type stars may form through disk-mediated accretion as their low-mass siblings do, but also show that the disk structure may be significantly perturbed by tidal interactions with (unseen) companions, even in a relatively poor cluster such as that associated with IRAS $20126+4104$.
\end{abstract}

Key words. stars: formation - ISM: jets and outflows - ISM: individual objects: IRAS 20126+4104

\section{Introduction}

The quest for disks around newly formed early-type stars is one of the challenges of modern astronomy. While there is general consensus that solar-type stars form through disk-mediated accretion, it is still debated whether a scaled-up version of the same formation mechanism holds for high-mass stars. The latter are commonly defined as those stars in excess of $\sim 6-8 M_{\odot}$ (Palla $\&$ Stahler 1993), a limit that for high accretion rates may become significantly higher (Hosokawa \& Omukai 2009). Stars like these reach the zero-age main sequence still undergoing accretion from their parental core. In this situation, the powerful radiation pressure of the star is expected to halt the infalling material, thus preventing further growth of the stellar mass. This socalled radiation pressure problem has recently found a solution that involves accretion through a circumstellar disk. The studies by Krumholtz et al. (2009) and Kuiper et al. $(2010,2011)$ have demonstrated that stars up to $140 M_{\odot}$ can be formed with this mechanism, and basically all current star formation models predict the existence of circumstellar disks around high-mass stars.

\footnotetext{
* Based on observations carried out with the Plateau de Bure interferometer.
}

The general consensus among theorists on the existence of disks around OB-type stars is in contrast with the lack of observational evidence. To date, only a handful of disk candidates are known, and basically all of these are associated with early B-type stars, whereas disks around the most massive, O-type stars appear elusive (Cesaroni et al. 2007). Very likely, this is due to the difficulty to resolve objects of $\$ 1000$ AU in clustered environments at large distances (typically a few kpc or more). Nonetheless, the important role that circumstellar disks could play in the high-mass star formation process makes such a lack of information very frustrating. While this situation is bound to change with the advent of the Atacama Large Millimeter Array (see results of Sánchez-Monge et al. 2013), it is nonetheless important to consolidate our knowledge of the few convincing examples of disks around B-type stars known to date. We therefore performed new high-angular resolution observations of the best candidate circumstellar disk around a high-mass protostars: IRAS 20126+4104

This object has been the subject of a long series of studies, which have provided evidence for the presence of a (presumably) Keplerian disk rotating about a $\sim 10^{4} L_{\odot}$ protostar and associated with a bipolar jet/outflow undergoing precession about the disk 
axis (Cesaroni et al. 1997, 1999, 2005; Keto \& Zhang 2010; Shepherd et al. 2000; Lebrón et al. 2009; Hofner et al. 1999, 2007; Caratti o Garatti et al. 2008). Multi-epoch VLBI observations of water masers (Moscadelli et al. 2005, 2011) have been used both to study the expansion of the jet and to determine the source distance with great accuracy $(1.64 \pm 0.05 \mathrm{kpc})$. More recently, adaptive optics images in the near-IR have permitted us to analyse the structure of the bipolar jet close to the deeply embedded protostar (Cesaroni et al. 2013, hereafter C2013).

Despite the progress made in our knowledge of this object, the structure of the disk and its relationship with the jet remain poorly known, because given the extinction of the dusty envelope around the protostar, only (sub)mm observations at subarcsecond resolution of a suitable molecular line can trace the structure and velocity field of the innermost region inside $~ 1000 \mathrm{AU}$ from the protostar. The interferometric observations performed by Cesaroni et al. (1997, 1999, 2005) were hindered by limited spectral and/or angular resolution, and could therefore only investigate the disk on a relatively large scale (>1000 AU), where the rotation speed is lower and hence more difficult to measure. With this in mind, we decided to take advantage of the capabilities of the Plateau de Bure interferometer to obtain new images of IRAS 20126+4104 in methyl cyanide, with unprecedented sensitivity and resolution. In this article we report on the results of these observations and shed light on the disk structure and its relationship with the associated bipolar jet on scales $\gtrsim 100$ AU.

\section{Observations and data reduction}

The data were collected in 2008 on January 29, February 13 and 16, and March 15 with the IRAM six-element array at Plateau de Bure, in the A and B array configurations. We used $2013+370$ and 3C 273 as phase and bandpass calibrators, while observations of MWC 349 were used to calibrate the flux density scale. The correlator was tuned at $220.218 \mathrm{GHz}$ in the lower side band, in such a way to cover all the $K$ components of the ground state and vibrationally excited $v_{8}=1 \mathrm{CH}_{3} \mathrm{CN}(12-11)$ transitions, as well as those of the $\mathrm{CH}_{3}{ }^{13} \mathrm{CN}$ isotopomer, but the $K=11$ line - although the highest energy lines were not detected. The phase centre of the observations was set at the coordinates $\left.\alpha(\mathrm{J} 2000)=20^{\mathrm{h}} 14^{\mathrm{m}} 26^{\mathrm{s}} .0364, \delta(\mathrm{J} 2000)=+41^{\circ} 13^{\prime} 32^{\prime \prime} .516\right)$.

The data were calibrated, reduced, and analysed with standard procedures, using the GILDAS package ${ }^{1}$. The typical phase noise was $\sim 30^{\circ}$. Channel maps with natural weighting over the whole frequency range covered were produced resulting in a synthesized half-power beam width (HPBW) of $0 .{ }^{\prime} 46 \times 0 . ' 32$ with position angle (PA) of $56^{\circ}$. The line-free channels were identified and used to produce continuum data which were then subtracted from the original data in the $u, v$ domain. Finally, continuum-subtracted cubes were created using natural weigthing. The conversion from flux density per beam to brightness temperature is given by the expression: $T_{\mathrm{B}}(\mathrm{K}) \simeq$ $169 S_{v}(\mathrm{Jy} /$ beam $)$.

\section{Results}

While a large number of lines from different molecules were detected in the broad bandwidth covered by our observations, in the following we will limit our analysis to the transitions of

\footnotetext{
The GILDAS software has been developed at IRAM and Observatoire de Grenoble - see http://www.iram.fr/IRAMFR/ GILDAS
}

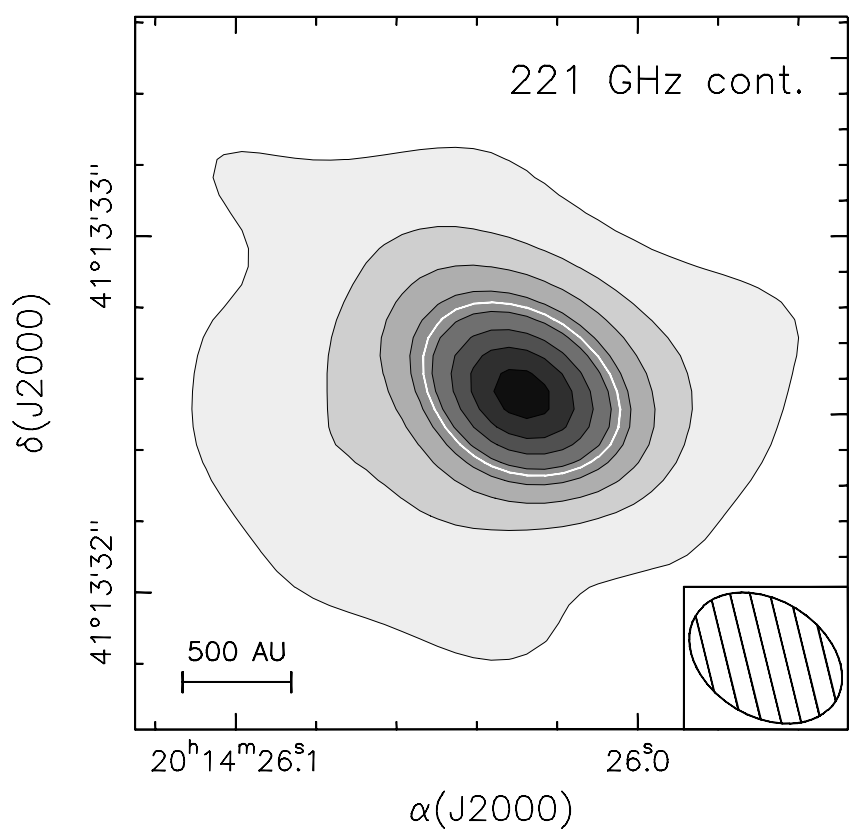

Fig. 1. Map of the $1.4 \mathrm{~mm}$ continuum emission towards IRAS 20126+ 4104. Contour levels range from 6 to $106.8 \mathrm{mJy} / \mathrm{beam}$ in steps of $14.4 \mathrm{mJy} /$ beam $(1 \sigma \mathrm{RMS} \simeq 1.5 \mathrm{mJy} / \mathrm{beam})$. The white contour corresponds to $50 \%$ of the peak flux density.

$\mathrm{CH}_{3} \mathrm{CN}$ and its ${ }^{13} \mathrm{C}$ substituted species $\mathrm{CH}_{3}{ }^{13} \mathrm{CN}$, as previous studies (Cesaroni et al. 1997, 1999) have proved these to be the best tools to investigate the disk. Also, the $\mathrm{CH}_{3} \mathrm{CN}$ lines span a large range of excitation energies and opacities, which allows us to sample different layers inside the disk.

\subsection{Continuum emission}

The continuum map obtained from the line-free channels is shown in Fig. 1. The integrated flux is $0.29 \mathrm{Jy}$, consistent with the millimetre continuum spectrum derived by Cesaroni et al. (2005, hereafter C2005; see their Fig. 14). From the figure one sees that most of the emission is contained in an almost unresolved structure, whose mean full width at half maximum (FWHM) is $\sim 0$.'5. Applying Gaussian deconvolution, one obtains an intrinsic angular diameter of $\sim 0$ ' 32 (525 AU) and a brightness temperature corrected for the beam filling factor of $\sim 47 \mathrm{~K}$. Since the maximum measured brightness temperature of the $\mathrm{CH}_{3} \mathrm{CN}$ lines is $\sim 100 \mathrm{~K}$ and the $\mathrm{CH}_{3} \mathrm{CN}$ rotational temperature is $\$ 200 \mathrm{~K}$ (see Cesaroni et al. 1997), the dust temperature must lie between these two extremes, which implies a dust opacity (along the line of sight through the disk centre) in the range $0.27-0.63$. Values like these suggest that the effect of dust absorption could be not entirely negligible even at this relatively long wavelength.

An optical depth of 0.27 at $231 \mathrm{GHz}$, corresponds to an $\mathrm{H}_{2}$ column density (through the disk centre) of $\sim 3 \times 10^{24}-3.2 \times$ $10^{25} \mathrm{~cm}^{-2}$, depending on the dust absorption coefficient adopted (ranging from $0.03 \mathrm{~cm}^{2} \mathrm{~g}^{-1}$ of Hildebrand et al. 1983 to $0.0025 \mathrm{~cm}^{2} \mathrm{~g}^{-1}$ of the Planck Collaboration XXI 2011). Such a large value is consistent with an edge-on disk and explains why the embedded protostar is not detected even at near-IR wavelengths (Sridharan et al. 2005; C2013). The presence of a prominent temperature and column density peak at the centre is consistent with the gas attaining its maximum temperature and density close to the embedded protostar, as expected. 


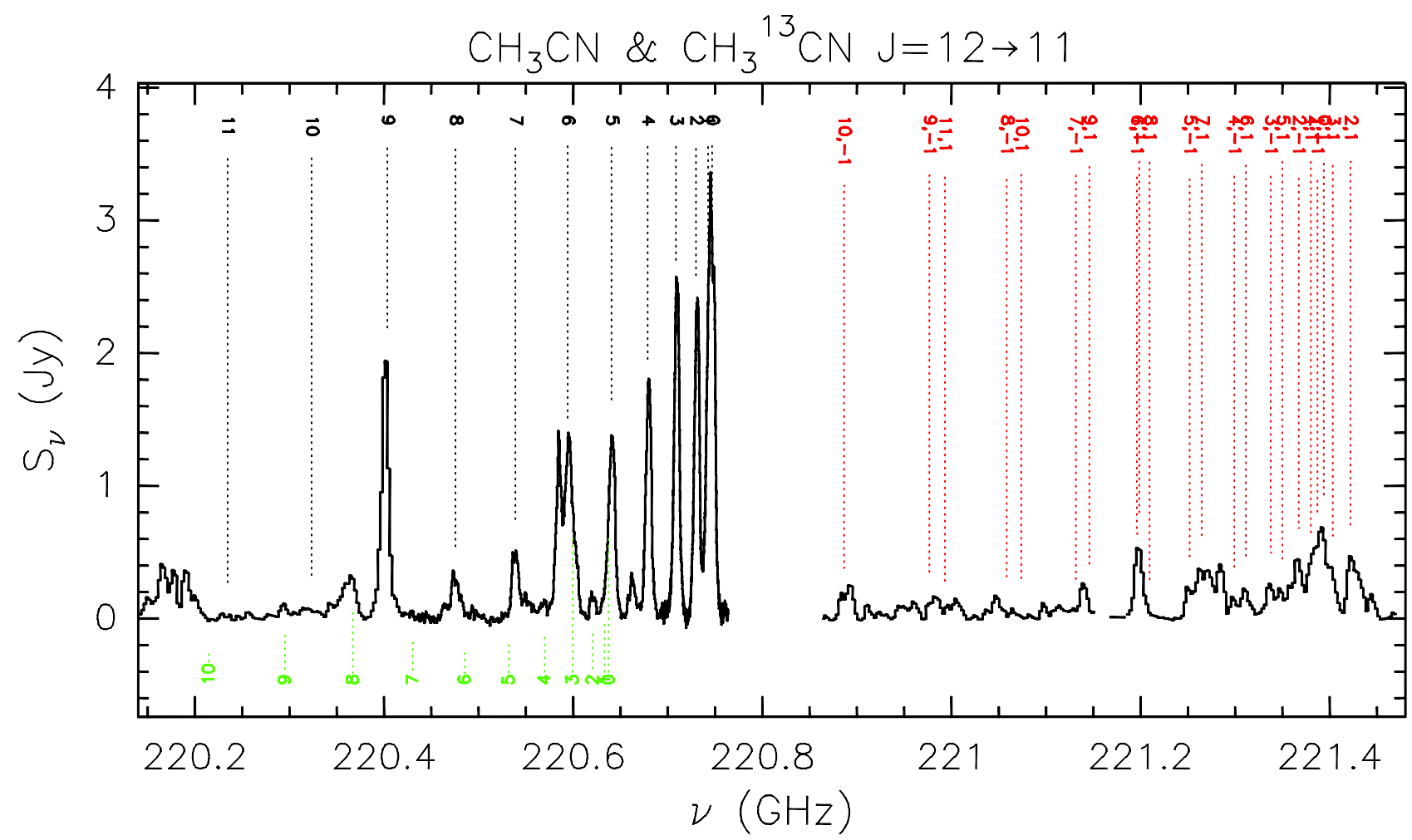

Fig. 2. Methyl cyanide spectrum obtained with the Plateau de Bure interferometer towards IRAS 20126+4104. The line emission has been integrated over the core. The dotted lines mark the $\mathrm{CH}_{3} \mathrm{CN}$ (from top) and $\mathrm{CH}_{3}{ }^{13} \mathrm{CN}$ (from bottom) transitions. The single numbers denote the $K$ component, while the number pairs correspond to the $K, l$ quantum numbers of the vibrationally excited, $v_{8}=1$, transitions. We note how most of the $\mathrm{CH}_{3} \mathrm{CN}$ and $\mathrm{CH}_{3}{ }^{13} \mathrm{CN}$ components are blended with other methyl cyanide lines and/or with lines of other species.

It is also worth noting that the compact continuum emission is surrounded by a much fainter halo slightly elongated to the $\mathrm{SE}$, i.e. in the direction of the red-shifted lobe of the jet/outflow (see e.g. C2005). This extension is also seen in the $\mathrm{CH}_{3} \mathrm{CN}$ lines, and will be discussed in Sect. 4.3.

\subsection{Line emission}

Figure 2 shows the spectrum obtained by integrating the emission over the region where methyl cyanide was detected (i.e. within $\sim 1^{\prime \prime}$ from the phase centre). Although this spectrum covers almost all of the ground state and $v_{8}=1$ transitions of $\mathrm{CH}_{3} \mathrm{CN}$, as well as those of $\mathrm{CH}_{3}{ }^{13} \mathrm{CN}$, only few lines are not blended. For this reason, unless otherwise specified, we will analyse only the components that do not overlap significantly with other transitions. These are the $K=2,3,4,8$ and $v_{8}=1$ $K, l=1,1$ components of $\mathrm{CH}_{3} \mathrm{CN}$, and the $K=2$ of $\mathrm{CH}_{3}{ }^{13} \mathrm{CN}$.

In Fig. 3 we show the profiles of the selected transitions, which appear slightly asymmetric and with broad wings. We associate the velocity interval from -10 to $+2 \mathrm{~km} \mathrm{~s}^{-1}$ with the bulk emission, beyond which the line wings become prominent. The maps in Fig. 4 have been obtained by averaging the line emission over this velocity interval. Inspection of the profiles reveals that the asymmetry changes with the energy and opacity of the transition. While the $K=2-4$ components appear more prominent in the blue-shifted emission, the $K=8$ and $K, l=1,1$ lines of $\mathrm{CH}_{3} \mathrm{CN}$ and the $K=2 \mathrm{CH}_{3}{ }^{13} \mathrm{CN}$ line are significantly skewed towards the red ${ }^{2}$. This velocity change may be quantified with

\footnotetext{
2 In the $K=8$ spectrum the bump of emission extending beyond the red wing (indicated by the arrow in Fig. 3) is likely due to the glycolaldehyde $20_{2,18}-19_{3,17}$ transition - detected for the first time by Beltrán et al. (2009) towards the hot molecular core G31.41+031.
}

the first moment computed in the interval -10 to $+2 \mathrm{~km} \mathrm{~s}^{-1}$, which is $-4.2,-4.1$, and $-3.9 \mathrm{~km} \mathrm{~s}^{-1}$ for the $K=2,3,4$ components, respectively, and $-3.3,-3.4$ and $-2.7 \mathrm{~km} \mathrm{~s}^{-1}$ for the other three lines. This behaviour is mirrored also in the maps of Fig. 4, where the peak of the emission appears to shift gradually from NE to SW for increasing excitation energy and decreasing opacity, until it coincides with the continuum peak, for the $\mathrm{CH}_{3} \mathrm{CN}$ $K, l=1,1$ and $\mathrm{CH}_{3}{ }^{13} \mathrm{CN} K=2$ transitions.

The previous findings suggest that the colder and possibly lower density gas (seen in the $\mathrm{CH}_{3} \mathrm{CN} K=2,3,4$ lines) is distributed asymmetrically with respect to the protostar (which should roughly coincide with the mm continuum peak), whereas the hotter gas is found inside a small region around the protostar. This interpretation implies that the disk is not axially symmetric. We will come back to this issue in Sect. 4.1.

Also worth of notice is the slight extension of the emission to the SE, alike to that observed for the continuum emission in Fig. 1. We consider this an indication that a minor fraction of the methyl cyanide emission could come from the red lobe of the bipolar outflow, which extends to the SE of the protostar (see e.g. Cesaroni et al. 1999). Such a result is not surprising, because evidence of $\mathrm{CH}_{3} \mathrm{CN}$ emission in molecular outflows has been found in other similar objects (see Leurini et al. 2011).

\section{Analysis and discussion}

\subsection{Disk structure}

An interesting feature revealed by the new $\mathrm{CH}_{3} \mathrm{CN}$ images is that the disk appears asymmetric with respect to the rotation axis passing through the continuum peak, which we assume to pin point the position of the star. This asymmetry has already 


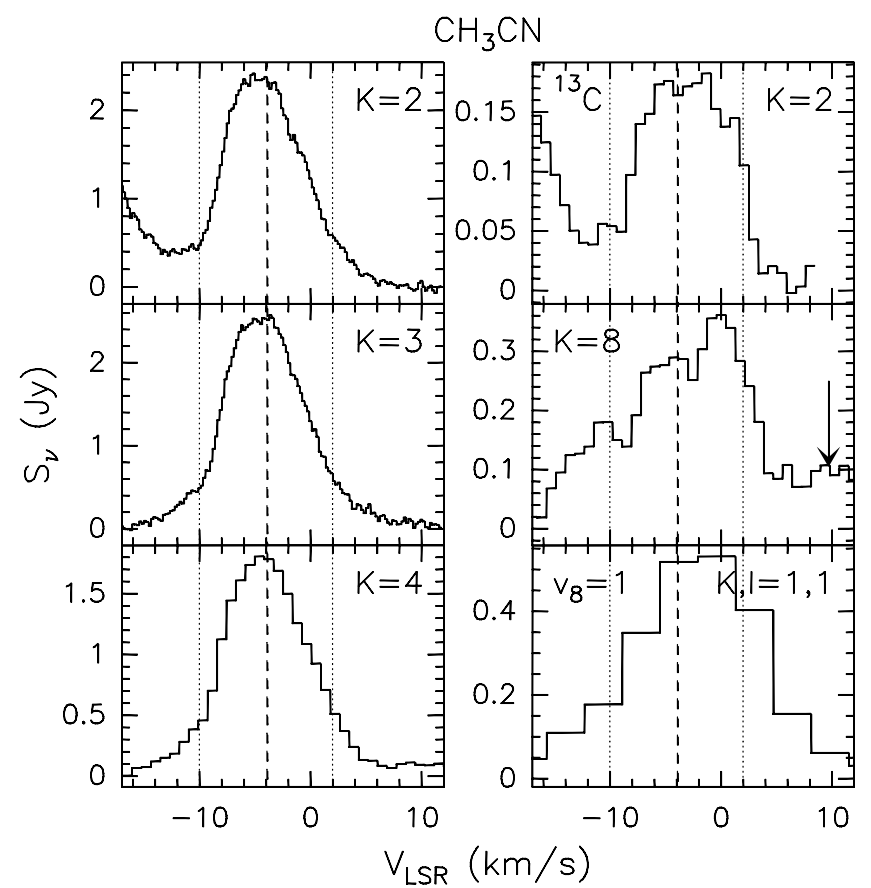

Fig. 3. Spectra of the $\mathrm{CH}_{3} \mathrm{CN}$ and $\mathrm{CH}_{3}{ }^{13} \mathrm{CN}$ transitions that are not significantly affected by blending with other lines. The vertical dashed line denotes the systemic velocity $\left(-3.9 \mathrm{~km} \mathrm{~s}^{-1}\right)$, while the dotted lines mark the beginning of the line wings. The arrow in the panel of the $K=8$ line marks the frequency of the $\mathrm{CH}_{2} \mathrm{OHCHO}\left(20_{2,18}-19_{3,17}\right)$ transition detected by Beltrán et al. (2009).

been noted in Sect. 3.2 on the basis of the line maps in Fig. 4. Asymmetries are not exceptional in lower mass sources, as demonstrated by the case of the Herbig star Oph IRS 48, where the material turns out to be irregularly distributed in the associated transitional disk (van der Marel et al. 2013, 2014; Bruderer et al. 2014). A method to emphasize our finding and better analyse the physical structure of the disk in IRAS 20126+4104, is to study the distribution of the line emission at different velocities. This can be obtained by fitting all channel maps of a given line with a 2D Gaussian, which provides us with an estimate of the peak position in each velocity channel. Figure 5 compares the distribution of the peaks for the $K=3$ component, with those of the $K=8$ and $K, l=1,1$ lines. Two considerations are in order. The first is that the distribution described by the peaks is significantly more extended to the NE than to the SW. The second is that the higher excitation transitions trace a smaller region than the $K=3$ line. The latter finding is consistent with a temperature increase towards the centre of the disk, as expected if the disk is centrally heated. This result is also consistent with previous studies of IRAS $20126+4104$, which, assuming a temperature variation with radius of $T \propto R^{q}$, obtain a negative power-law index ranging from $q \lesssim-1$ (Keto \& Zhang 2010) to $q \simeq-0.57$ (C2005). While for a geometrically thin disk one expects $q=-0.75$ (Pringle 1981), the presence of an envelope in IRAS 20126+4104 and the non-negligible geometrical thickness of the disk (the hydrostatic scale height ${ }^{3}$ is $z 430 \mathrm{AU}$ ) result in a flatter $(q>-0.75)$ temperature profile (see e.g. Chiang $\&$ Goldreich 1997). Better angular resolution and modelling are

\footnotetext{
3 The scale height, $H$, has been computed from the expression $H=(F W H M / \sqrt{8 \ln 2}) \sqrt{R_{\text {disk }}^{3} /\left(G M_{\text {star }}\right)}$ obtained by replacing $c_{\mathrm{s}}$ with $F W H M / \sqrt{8 \ln 2}$ in Eq. (3.14) of Pringle (1981) and assuming $F W H M \simeq$ $3 \mathrm{~km} \mathrm{~s}^{-1}, M_{\text {star }} \lesssim 10 M_{\odot}$, and $R_{\text {disk }} \simeq 1000 \mathrm{AU}$.
}

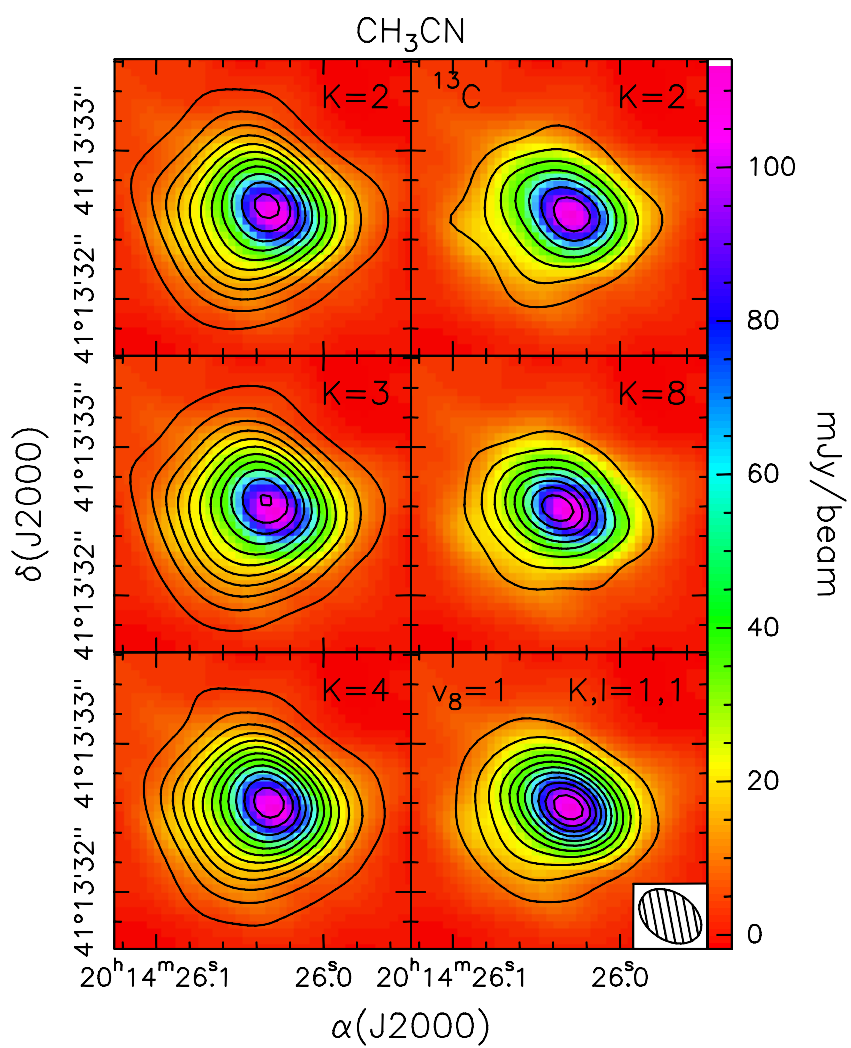

Fig. 4. Contour maps of the bulk emission in the same lines as in Fig. 3, obtained by averaging the emission over the velocity interval delimited by the dotted lines in Fig. 3. The background image is the continuum emission shown also in Fig. 1. Contour levels range: from 21.6 to 381.6 in steps of $36 \mathrm{mJy} /$ beam $(1 \sigma \mathrm{RMS} \simeq 7 \mathrm{mJy} / \mathrm{beam})$ for the $K=2$ line; from 22.8 to 402.8 in steps of $38 \mathrm{mJy} /$ beam $(1 \sigma$ RMS $\simeq 8 \mathrm{mJy} / \mathrm{beam})$ for the $K=3$; from 16.8 to 324.8 in steps of $28 \mathrm{mJy} / \mathrm{beam}(1 \sigma \mathrm{RMS} \simeq$ $6 \mathrm{mJy} /$ beam) for the $K=4$; from 4.5 to 49.5 in steps of $7.5 \mathrm{mJy} / \mathrm{beam}$ $\left(1 \sigma \mathrm{RMS} \simeq 1.5 \mathrm{mJy} /\right.$ beam) for the $K=2$ line of $\mathrm{CH}_{3}{ }^{13} \mathrm{CN}$; from 8.7 to 95.7 in steps of $14.5 \mathrm{mJy} / \mathrm{beam}(1 \sigma \mathrm{RMS} \simeq 3 \mathrm{mJy} / \mathrm{beam})$ for the $K=8$; and from 8.7 to 153.7 in steps of $14.5 \mathrm{mJy} /$ beam $(1 \sigma \mathrm{RMS} \simeq$ $3 \mathrm{mJy} /$ beam) for the $K, l=1,1$ line.

required to derive a precise estimate of the temperature across the disk.

As for the shorter extent of the disk to the SW, one may wonder whether this might be due to truncation by a nearby companion, perhaps associated with the thermal jet detected by Hofner et al. $(1999,2007)$ about $0 .^{\prime} 8$ to the south of IRAS $20126+4104$. A rough estimate of the companion mass $M_{\mathrm{c}}$ needed to cause such a truncation is obtained assuming that the radius of the disk is equal to the radius of the gravitational sphere of influence of the protostar (the Hill radius). From e.g. Eq. (1) of Lissauer \& Stevenson (2007), one obtains

$M_{\mathrm{c}} \simeq \frac{M_{*}}{3}\left(\frac{d_{\mathrm{c}}}{R_{\mathrm{d}}}\right)^{3}$,

where $M_{\mathrm{c}}$ is the mass of the companion, $M_{*} \simeq 7 M_{\odot}$ that of the protostar, $R_{\mathrm{d}} \simeq 0.2$ is the length of the radius to the $\mathrm{SW}$ of the protostar (see Fig. 5), and $d_{\mathrm{c}}$ is the distance of the companion from the protostar. A lower limit to the latter is the projected offset of $\sim 0$ '. $^{\prime} 8$ (see above). From Eq. (1) one thus obtains a lower limit $M_{\mathrm{c}}>150 M_{\odot}$, too large to be credible.

Perhaps it is more likely that the disk truncation is caused by a nearby, unseen, low-mass companion, located very close to the border of the disk to the SW. Assuming $d_{\mathrm{c}} \simeq R_{\mathrm{d}}$ in Eq. (1), one 


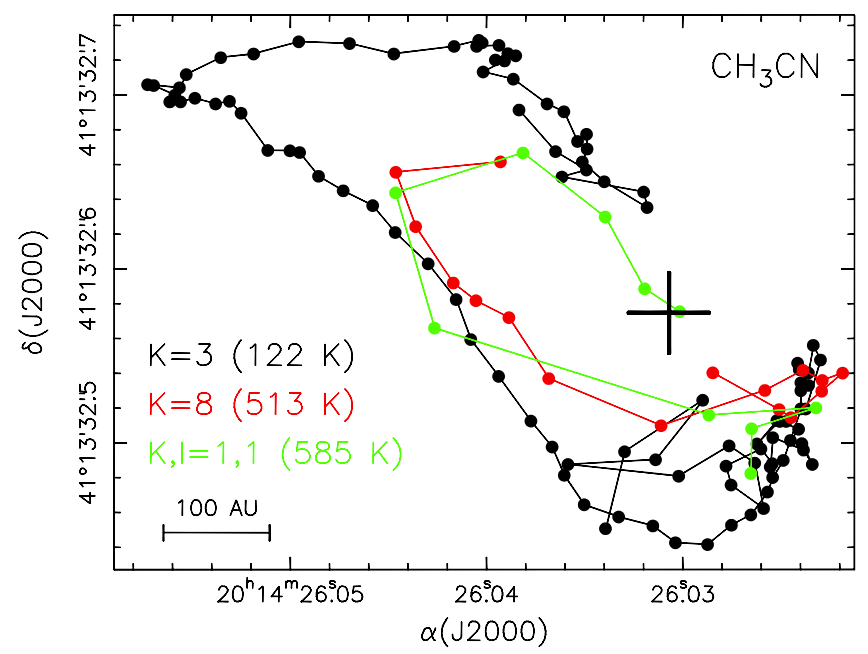

Fig. 5. Distributions of the $\mathrm{CH}_{3} \mathrm{CN}$ emission peaks at different velocities, obtained with a 2D Gaussian fit to the channel maps of the $K=3$ (black points), $K=8$ (red), and $K, l=1,1$ (green) lines. The cross marks the position of the continuum peak, which we believe to be a good approximation for the location of the protostar. We note how the higher excitation lines trace a region closer to the protostar.

gets $M_{\mathrm{c}} \simeq 2 M_{\odot}$. As a matter of fact, the presence of a binary system in IRAS 20126+4104 is suggested by the precession of the jet/outflow, as discussed by Shepherd et al. (2000).

Another possibility is that the southern jet is sweeping away the material from the disk, thus causing the observed NE-SW asymmetry, but we cannot exclude that such an asymmetrical distribution of material might be caused by instabilities in the plane of the disk, as suggested by the results illustrated later on in Sect. 4.2.

While these and other hypotheses are purely speculative at the moment, one consideration is in order. Should the disk around IRAS $20126+4104$ be affected by any interaction with nearby companions, this would be surprising, because the cluster associated with IRAS $20126+4104$ is significantly less populated than the clusters around similar young stellar objects (see Qiu et al. 2008). This result might contain a lesson to learn: if even a poorly populated region can significantly affect the disk structure around a B-type protostar, such an effect should be even more dramatic for disks around O-type (proto)stars, (known to lie inside rich stellar clusters) and could explain why these disks are so elusive (see Sect. 1).

\subsection{Disk velocity field}

In their study, C2005 have presented evidence that the disk around IRAS $20126+4104$ is undergoing Keplerian rotation about a $\sim 7 M_{\odot}$ star, up to a radius of at least $\sim 0.03 \mathrm{pc}$. This conclusion was reached by means of observations of two rotational transitions of $\mathrm{C}^{34} \mathrm{~S}$, which is sensitive to relatively low densities $\left(\sim 10^{6} \mathrm{~cm}^{-3}\right)$ and traces only the outer, slowly rotating part of the disk. A similar conclusion was reached by Keto \& Zhang (2010), who fitted the $\mathrm{CH}_{3} \mathrm{CN}$ and $\mathrm{NH}_{3}$ line profiles at selected positions across the disk with a numerical model assuming the presence of both a disk and a surrounding envelope undergoing rotation and infall. Their best fit implies a stellar mass of $10.7 M_{\odot}$, but values as small as $8.3 M_{\odot}$ cannot be ruled out (see their Fig. 9). Our new data allow us to improve on the previous results, because the $\mathrm{CH}_{3} \mathrm{CN}(12-11)$ lines arise from a denser, hotter, and hence

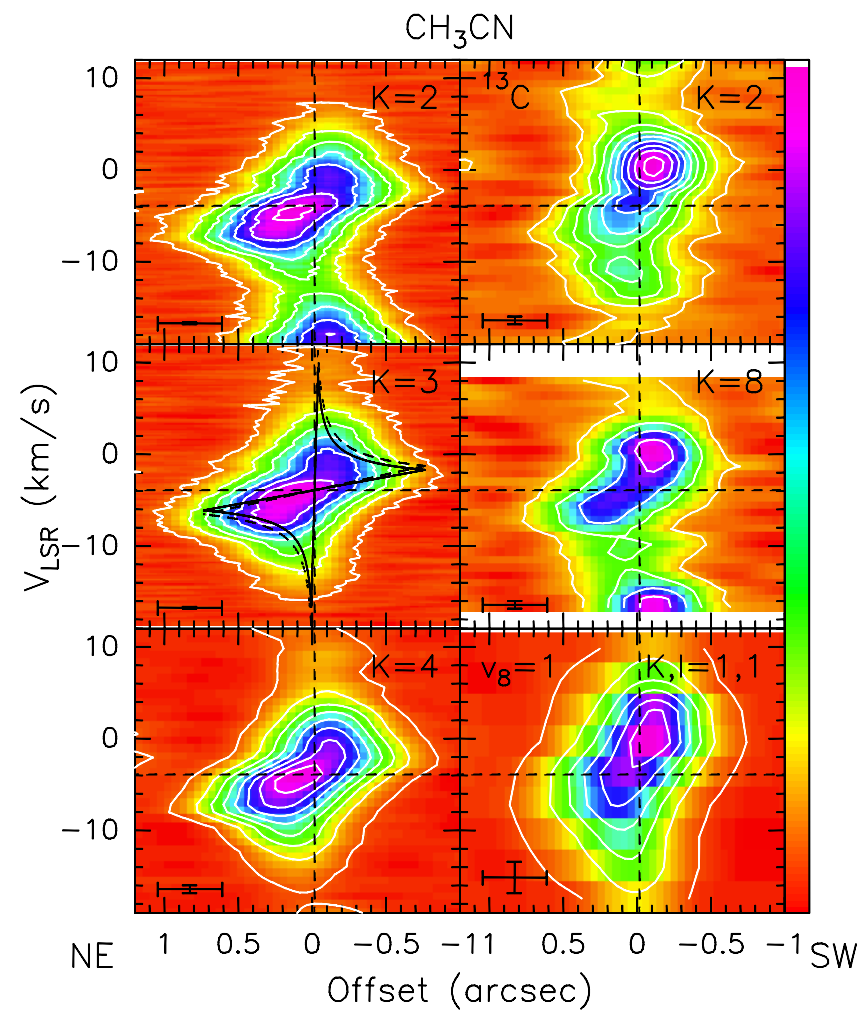

Fig. 6. Position-velocity plots along a direction with $\mathrm{PA}=+37^{\circ}$ passing through the continuum peak. The crosses in the bottom left corners denote the spatial and spectral resolutions. Contour levels (in units of brightness temperature in the synthesized beam) range: from 4 to 82 in steps of $13 \mathrm{~K}(1 \sigma \mathrm{RMS} \simeq 1.2 \mathrm{~K})$ for the $K=2$ line; from 4 to 74 in steps of $14 \mathrm{~K}(1 \sigma \mathrm{RMS} \simeq 1.2 \mathrm{~K})$ for the $K=3$; from 3 to 66 in steps of $9 \mathrm{~K}(1 \sigma \mathrm{RMS} \simeq 0.67 \mathrm{~K})$ for the $K=4$; from 2 to 26 in steps of $3 \mathrm{~K}$ $(1 \sigma \mathrm{RMS} \simeq 0.42 \mathrm{~K})$ for the $K=2$ line of $\mathrm{CH}_{3}{ }^{13} \mathrm{CN}$; from 1 to $10 \mathrm{in}$ steps of $3 \mathrm{~K}(1 \sigma \mathrm{RMS} \simeq 0.61 \mathrm{~K})$ for the $K=8$; and from 1 to 26 in steps of $5 \mathrm{~K}(1 \sigma \mathrm{RMS} \simeq 0.24 \mathrm{~K})$ for the $K, l=1,1$ line. The dashed horizontal and vertical lines correspond to the systemic velocity and position of the $1.4 \mathrm{~mm}$ continuum peak. The "butterfly-shaped" patterns in the $K=3$ panel outline the regions inside which emission is expected if the gas lies in a disk with radius of 0.75 ( $\sim 1200 \mathrm{AU})$, rotating about a $7 M_{\odot}$ star (solid pattern) and a $10 M_{\odot}$ star (dashed).

smaller region, where the gravitational field should be dominated by the protostar.

To investigate the disk velocity field we have produced position-velocity (PV) plots analogous to those presented by C2005 (see their Fig. 7). These are shown in Fig. 6 and refer to a cut along a direction with $\mathrm{PA}=+37^{\circ}$ passing through the continuum peak. In order to improve the signal to noise of the plots, we also averaged the emission along the direction perpendicular to the cut. We note that this average does not include the $\mathrm{CH}_{3} \mathrm{CN}$ emission extending to the $\mathrm{SE}$, because this is likely associated with the outflow - as discussed in Sect. 3.2.

For the sake of comparison, we overlay on the PV plot of the $K=3$ line, the patterns encompassing the regions from which emission is expected in the cases of a Keplerian disk rotating about a $7 M_{\odot}(\mathrm{C} 2005)$ and $10 M_{\odot}$ (Keto \& Zhang 2010) star. Clearly, these are not intended to fit the data (as they do not consider the line width and the limited angular resolution), but have the sole purpose to outline the "butterfly-shaped" pattern of the emission, typical of rotation curves with velocity $\left(V_{\text {rot }}\right)$ increasing towards the centre. A special case is that of Keplerian rotation, where $V_{\text {rot }} \propto R^{-1 / 2}$. Here, a caveat is in order. Both C2005 and Keto \& Zhang (2010) obtained a satisfactory fit to 


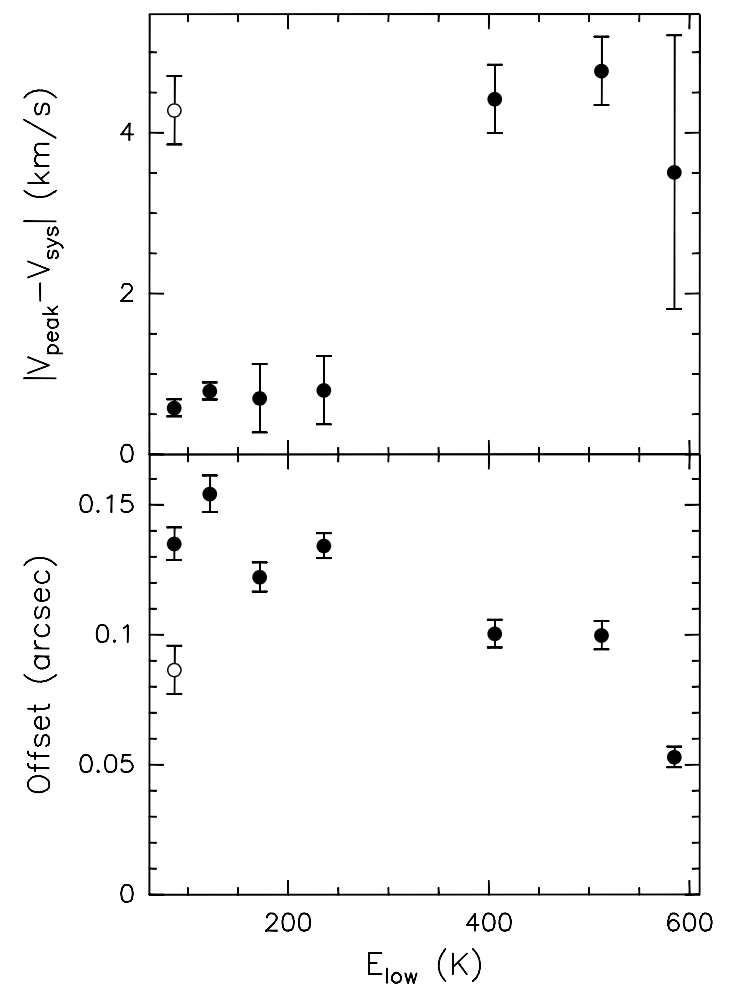

Fig. 7. Plots of the peak positions in the PV plots in Fig. 6 versus the energy of the lower level of the corresponding transition. The black points correspond, from left to right, to the $K=2,3,4,5,7,8$ and $v_{8}=1$ $K, l=1,1$ lines, while the white point denotes the $\mathrm{CH}_{3}{ }^{13} \mathrm{CN} K=2$ line. Top: absolute value of the relative velocity of the peak with respect to the systemic velocity, $V_{\text {sys }} \simeq-3.9 \mathrm{~km} \mathrm{~s}^{-1}$. The bars correspond to half the velocity channel. Bottom: absolute value of the distance of the peak from the position of the continuum peak, measured along the plane of the disk (i.e. along the direction with $\mathrm{PA}=+37^{\circ}$ ).

the data, assuming a priori a velocity field for the disk (+envelope), but neither study demonstrates that Keplerian rotation is the best possible rotation curve. Thus, other laws of the type $V_{\text {rot }} \propto R^{\alpha}$ with $\alpha<0$ and $\alpha \neq-1 / 2$ might give a better fit, as in the case of the Herbig Ae star AB Aur where significant deviations from Keplerian rotation have been found (Pietu et al. 2005; Tang et al. 2012). With this in mind, in the following we will refer to the velocity field of the disk in IRAS 20126+4104 as (quasi-)Keplerian rotation.

In the PV plots, the asymmetry of the emission in the plane of the disk shows up more remarkably than in Figs. 3 and 4. Clearly, the transitions with the highest opacities and lowest excitations present a peak of emission to the NE, at low velocities $\left(\sim 1 \mathrm{~km} \mathrm{~s}^{-1}\right.$ relative to the systemic velocity), whereas at higher energies and lower optical depths the emission peak moves to the SW at significantly higher speeds $\left(\gtrsim 4 \mathrm{~km} \mathrm{~s}^{-1}\right)$. Figure 7 illustrates this effect, by reporting the velocity $\left(V_{\text {peak }}\right)$ and position of the peak in each PV plot versus the excitation energy of the corresponding transition. The positions have been obtained with a 2D Gaussian fit to the emission in the channel corresponding to $V_{\text {peak }}$ and the error has been computed from Eq. (1) of Reid et al. (1988). The velocity in Fig. 7 is computed relative to the systemic velocity (in absolute value) i.e. relative to the protostar, while the offset is the projected distance (in absolute value) from the continuum peak, which best approximates the stellar position. We note that to sample as many energy levels as possible, we have used also the $K=5$ and 7 components, as these are only partially overlapped to $\mathrm{CH}_{3}{ }^{13} \mathrm{CN}$ lines. The
Table 1. Velocity components along the line of sight and positions in the plane of the disk, of the peaks in the PV plots of various $\mathrm{CH}_{3} \mathrm{CN}$ and $\mathrm{CH}_{3}{ }^{13} \mathrm{CN}$ lines.

\begin{tabular}{lrrr}
\hline \hline$J=12-11$ transition & $V\left(\mathrm{~km} \mathrm{~s}^{-1}\right)$ & $x(\mathrm{AU})$ & $y(\mathrm{AU})$ \\
\hline $\mathrm{CH}_{3} \mathrm{CN} K=2$ & -0.26 & 221 & -943 \\
$\mathrm{CH}_{3} \mathrm{CN} K=3$ & -0.35 & 253 & -823 \\
$\mathrm{CH}_{3} \mathrm{CN} K=4$ & -0.31 & 200 & -774 \\
$\mathrm{CH}_{3} \mathrm{CN} K=5$ & -0.36 & 220 & -747 \\
$\mathrm{CH}_{3} \mathrm{CN} K=7$ & 1.96 & -165 & -122 \\
$\mathrm{CH}_{3} \mathrm{CN} K=8$ & 2.12 & -164 & -105 \\
$\mathrm{CH}_{3} \mathrm{CN} v_{8}=1 K, l=1,1$ & 1.56 & -87 & -130 \\
$\mathrm{CH}_{3}{ }^{13} \mathrm{CN} K=2$ & 1.90 & -142 & -126 \\
\hline
\end{tabular}

Notes. The velocities are computed with respect to the systemic velocity and the positional offsets are relative to the protostar (a distance of $1.64 \mathrm{kpc}$ has been assumed to calculate the linear offsets).

$K=6$ component is unusable because heavily blended with the $\operatorname{HNCO}\left(10_{19}-9_{18}\right)$ line.

Our findings support the reasonable expectation that the lower energy lines are emitted farther away from the star than the higher-excitation and/or lower-opacity transitions, because the latter are seen at larger offsets and lower velocities than the former. One can obtain a more quantitative estimate of the locations where the different lines are emitted. For the sake of simplicity, in the following we assume that the disk is undergoing pure Keplerian rotation. While the results may be quantitatively different for quasi-Keplerian rotation, the qualitative conclusions obtained below will not change significantly. In a Keplerian disk, the component of the rotational velocity along the line of sight is given by the expression

$V=\frac{x}{\left(x^{2}+y^{2}\right)^{\frac{3}{4}}}$,

where $V$ is normalized with respect to the rotational speed at a given radius, $x$ is the impact parameter normalized with respect to that radius, and $y$ is the coordinate along the line of sight, also normalized with respect to the same radius. From this, one can trivially obtain the value of $y$ as

$y= \pm \sqrt{\left(\frac{x^{2}}{V^{2}}\right)^{\frac{2}{3}}-x^{2}}$

where we assume that the negative solution corresponds to the part of the disk facing the observer.

Using the values of the impact parameters and velocities in Fig. 7 and assuming a disk radius of 0.75 and a corresponding velocity of $2.25 \mathrm{~km} \mathrm{~s}^{-1}$ (i.e. a central star of $\sim 7 M_{\odot}-$ see the Keplerian pattern in Fig. 6), one can compute the values of $y$ and hence locate the positions in the plane of the disk, where the various lines are emitted. These are listed in Table 1 and drawn in Fig. 8, where a sketch of the disk seen face on is presented. We have arbitrarily adopted the negative solution in Eq. (3). For the low- $K$ lines this choice is justified because the emission from the farthest point $(y>0)$ along the line of sight (1.o.s.) is likely to be self-absorbed at the closest point (at $y<0$ ), due to the large line opacity ${ }^{4}$. For the $\mathrm{CH}_{3}{ }^{13} \mathrm{CN}$ and higher-excitation lines our assumption is more questionable, as they are likely optically thin.

4 The mean optical depth over the $\mathrm{CH}_{3} \mathrm{CN}(12-11) \mathrm{K}=2$ line, estimated from the ratio to the same $\mathrm{CH}_{3}{ }^{13} \mathrm{CN}$ transition, is $\sim 7$, assuming an isotopic abundance ratio $\left[{ }^{12} \mathrm{C} /{ }^{13} \mathrm{C}\right]=76$ (Wilson \& $\operatorname{Rood} 1994$ ). 


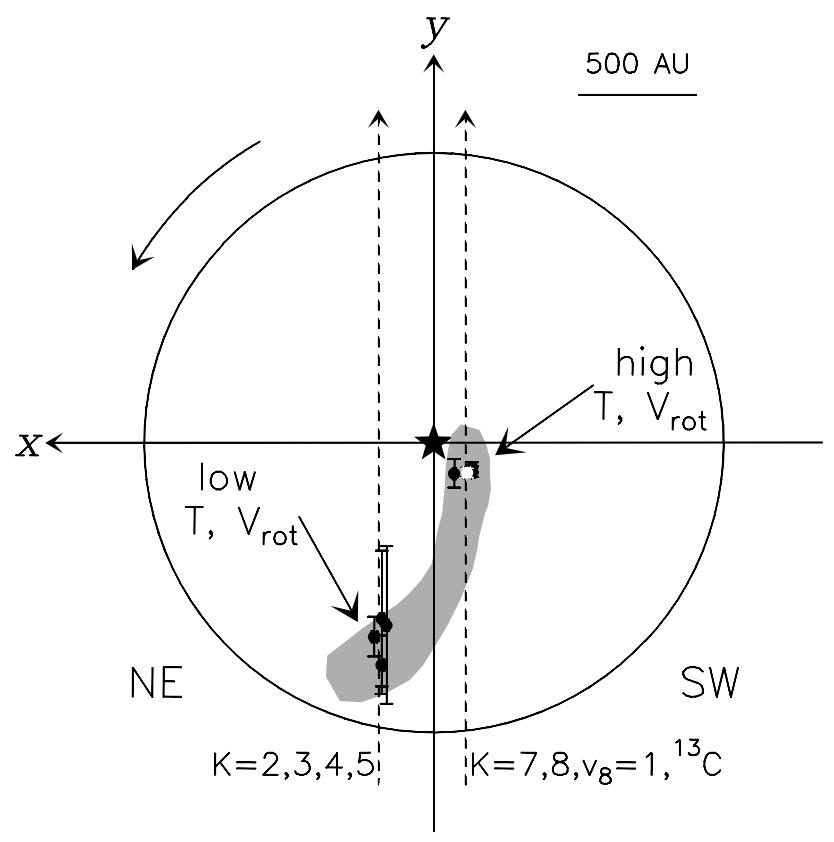

Fig. 8. $\mathrm{CH}_{3} \mathrm{CN}$ disk around IRAS 20126+4104. The observer lies at $y=$ $-\infty$ and sees the disk edge on. The curved arrow indicates the sense of rotation. The points mark the estimated positions of the same peaks as in Fig. 7, and colour-coded in the same way. The grey area outlines a tentative trailing spiral arm. The starred symbol marks the position of the protostar and the two dashed lines are two lines of sight crossing the regions where the low- and high-energy lines peak. The two solid arrows indicate the two regions where the observed lines are emitted.

However, the corresponding points lie very close to the plane of the sky (i.e. at $y \simeq 0$; see Fig. 8 ), so the choice of the sign is quite unimportant.

The distribution of the points in Fig. 8 confirms that the lower energy lines are mostly arising in the outer region of the disk to the NE, whereas the higher excitation/lower optical depth transitions come from the inner region seen to the SW.

These results provide clear evidence of an asymmetric distribution of material in the disk. The question is whether such an asymmetry is due to inhomogeneities randomly distributed across the disk or instead mirrors a specific phenomenon strictly related to the accretion process and/or the dynamics of the disk itself. Clearly, the data available to us do not permit us to answer this question, but we can provide the reader with some speculations.

One possibility is that we are observing a spiral arm, as tentatively depicted in Fig. 8. The existence of spiral density structures is indeed predicted by numerical calculations and appears to become more prominent for increasing stellar masses (see e.g. Cossins et al. 2009, Kratter et al. 2010). We therefore believe that this possibility in the case of IRAS 20126+4104 cannot be disregarded. Alternatively, density enhancements might be provoked by material accreting onto the disk and, eventually, the protostar, alike to the infalling filaments observed by Tobin et al. (2012) in a number of low-mass young stellar objects. It is reasonable to expect that the interactions between the infalling envelope and the circumstellar disk are more prominent in the deeply embedded high-mass protostars than in their lowmass siblings. Finally, one cannot exclude that the disk structure may be affected by nearby companions, as already discussed in Sect. 4.1. These could generate instabilities and even open gaps in the disk, thus breaking the axial symmetry.

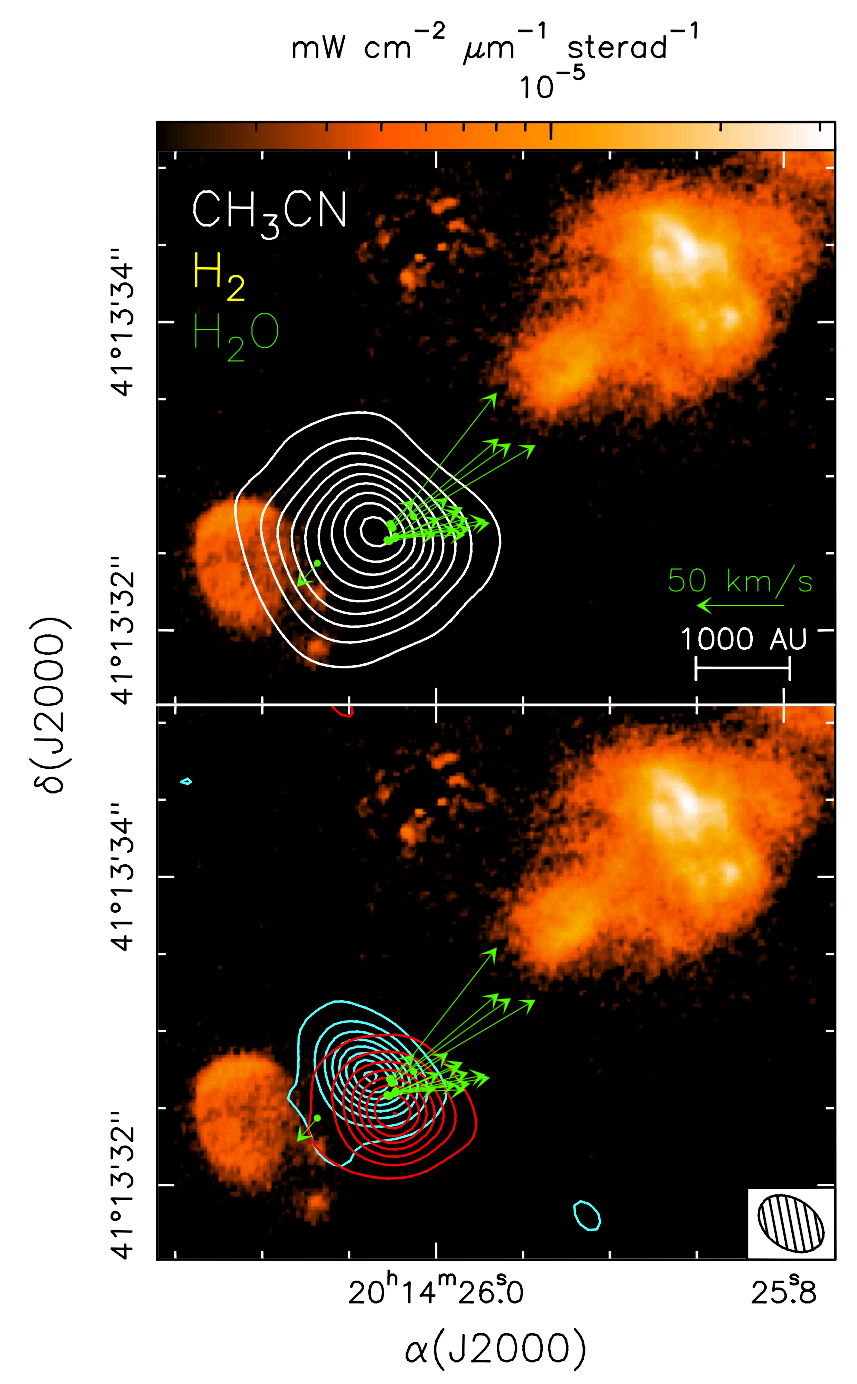

Fig. 9. Overlay of the bulk (top panel) and blue- and red-shifted emission (bottom panel) of the $\mathrm{CH}_{3} \mathrm{CN} \mathrm{K}=3$ line on the image of the $\mathrm{H}_{2}$ $2.2 \mu \mathrm{m}$ line emission obtained by $\mathrm{C} 2013$. The green points and arrows denote the $\mathrm{H}_{2} \mathrm{O}$ maser spots and associated proper motions measured by Moscadelli et al. (2005). Contour levels in the top panel are the same as in Fig. 4, while in the bottom panel range from 15 to 225 in steps of $30 \mathrm{mJy} /$ beam.

It is worth noting that, to some extent, our case resembles that of the lower-mass, Herbig Ae star AB Aur, where evidence for the existence of residual accretion, disk asymmetries, and spiral arms has been found (see Tang et al. 2012). However, the linear resolution attained in our case is an order of magnitude worse than that of the $\mathrm{AB}$ Aur observations, so that any comparison between the two objects is to be considered with caution. Higher resolution $\left(\lesssim 00^{\prime} 1\right)$ images are needed to draw any firm conclusion and discriminate between the different hypotheses to explain the observed asymmetrical distribution of the gas in the plane of the disk of IRAS $20126+4104$.

\subsection{The disk-jet connection}

Since one of the goals of the present study is to investigate the relationship between the disk and the associated jet/outflow, we compare the $\mathrm{CH}_{3} \mathrm{CN}$ emission to the near-IR images of C2013, which provide us with excellent information on the jet structure close to the protostar. This is done in Fig. 9, where both the bulk (top panel) and the blue- and red-shifted $\mathrm{CH}_{3} \mathrm{CN} \mathrm{K}=3$ emission 


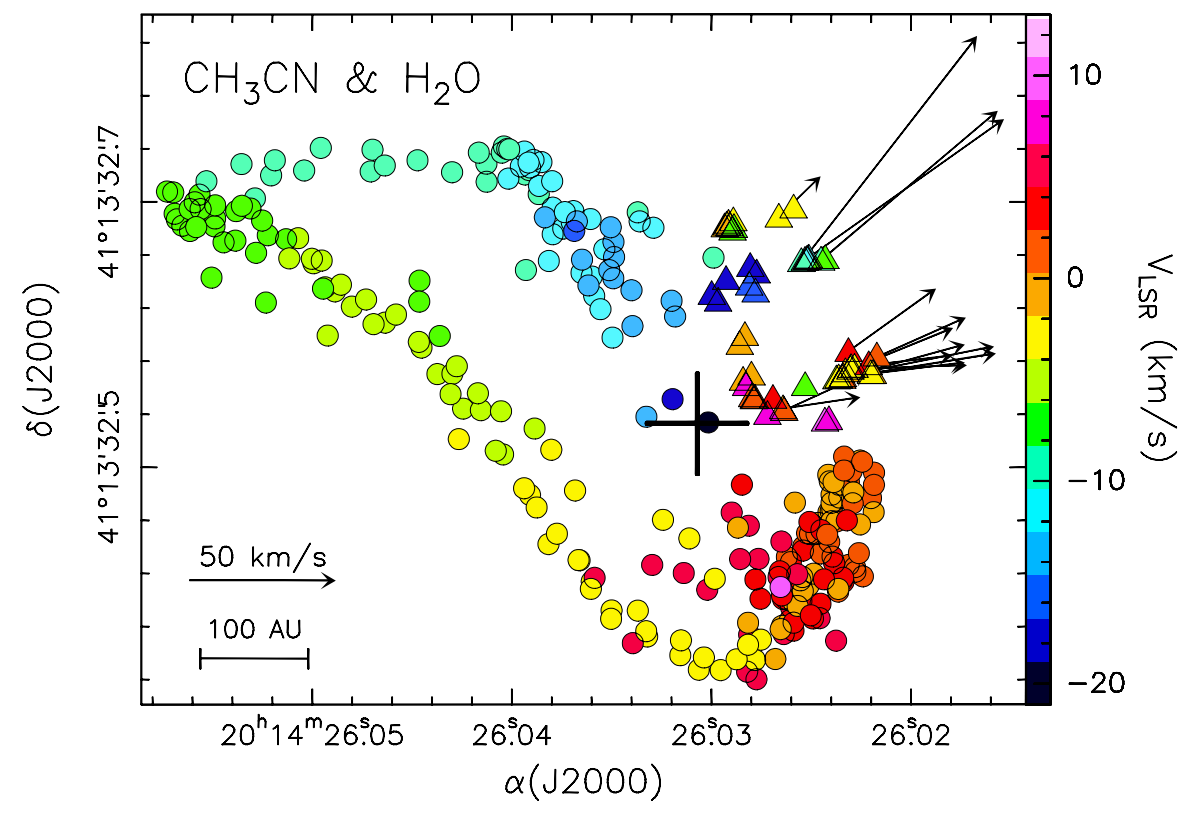

Fig. 10. Map of the peaks of the $\mathrm{CH}_{3} \mathrm{CN} K=$ 2,3, 4 line emission (circles) and water maser spots (triangles) from Moscadelli et al. (2000, 2005) and Edris et al. (2005). The colour indicates the LSR velocity according to the code reported in the scale to the right. The arrows indicate the proper motions measured by Moscadelli et al. (2005). The cross marks the position of the continuum emission peak. are overlaid on an image of the $2.2 \mu \mathrm{m} \mathrm{H}_{2}$ line emission. In the same figure, we also plot the proper motions of the water masers measured by Moscadelli et al. (2005).

Cesaroni et al. (2013) propose that the ejection episodes from the protostar occur at different times in the SE and NW lobes. In fact, the mushroom-shaped $\mathrm{H}_{2}$ structure to the NW lies at a larger distance from the protostar than the bubble of $\mathrm{H}_{2}$ emission seen to the SE. The interpretation of C2013 is that the latter corresponds to a more recent ejection episode than the former. The $\mathrm{H}_{2} \mathrm{O}$ maser spots expanding to the NW are likely associated with an even younger ejection, still piercing the surrounding envelope. This explains why no IR emission is seen close to the masers. Our data appear to confirm the scenario depicted by $\mathrm{C} 2013$, because the $\mathrm{CH}_{3} \mathrm{CN}$ contours are slightly extended towards the SE bubble, as if the emission were tracing the walls of the cavity encompassing the SE jet lobe. This opens up farther away from the star allowing the near-IR photons to escape and form the observed bubble. On the opposite side, the $\mathrm{CH}_{3} \mathrm{CN}$ emission drops sharply, right where the $\mathrm{H}_{2} \mathrm{O}$ masers appear. This suggests that $\mathrm{CH}_{3} \mathrm{CN}$ is not excited beyond a few $100 \mathrm{AU}$ to the NW of the protostar, possibly because of the dense, cold material against which the NW lobe is colliding.

It is believed that the expansion of the jets from low-mass (proto)stars could be driven by a mechanism involving a magnetic field corotating with the circumstellar disk. Indeed, in some cases evidence of jet rotation about its axis has been found (see e.g. Coffey et al. 2007). It is therefore of interest to find out whether this might be the case also for high-mass (proto)stars. Moreover, the existence of jet rotation would be strong evidence in favour of the existence of a disk rotating about the jet axis.

With this in mind, we have compared the velocity field of the disk around IRAS 20126+4104 with that of the associated jet, within a few $100 \mathrm{AU}$ from the surface of the disk itself. For this purpose, we have used the results of the 2D Gaussian fit described above. This is illustrated in Fig. 10, where the peaks of the $K=2,3,4$ components are shown as circles, with colour corresponding to the velocity associated with each peak. As expected for (quasi-)Keplerian rotation, the highest velocities are attained close to the disk centre, i.e. close to the protostar, whose position reasonably corresponds to the continuum peak. The only tracer that can be used to study the velocity field of the jet on the same scale are the $\mathrm{H}_{2} \mathrm{O}$ masers imaged with the VLBI by Moscadelli et al. $(2000,2005)$ and with MERLIN by Edris et al. (2005). These are plotted as triangles in the same figure ${ }^{5}$.

The maser 3D velocities are dominated by an expansion component along the jet of the order of $\sim 50-100 \mathrm{~km} \mathrm{~s}^{-1}$, which affects also the observed velocity along the l.o.s.. Therefore, if we want to unveil a possible rotational component of the jet, the expansion component must be subtracted from the maser 1.o.s. velocities. If one calculates the mean velocity along the 1.o.s. of all the $\mathrm{H}_{2} \mathrm{O}$ maser spots $\left(V_{\text {mean }} \simeq-9.4 \mathrm{~km} \mathrm{~s}^{-1}\right)$, the resulting azimuthal component should be zero because for each spot rotating towards the observer, ideally there should be another spot rotating away from it. In contrast, the expansion velocities are all very similar because the jet is very collimated, and hence their mean value is a good approximation to the effective LSR velocity of each spot due only to the expansion. The effective expansion speed along the l.o.s. is then obtained as $V_{\text {exp }}=V_{\text {mean }}-V_{\text {sys }} \simeq-5.5 \mathrm{~km} \mathrm{~s}^{-1}$, where $V_{\text {sys }} \simeq-3.9 \mathrm{~km} \mathrm{~s}^{-1}$ is the systemic velocity of IRAS $20126+4104$. Finally, $V_{\exp }$ was subtracted from the LSR velocities of the maser spots.

The result is shown in Fig. 10, where the same colour coding has been used for both the LSR velocities of the $\mathrm{CH}_{3} \mathrm{CN}$ peaks and those of the $\mathrm{H}_{2} \mathrm{O}$ maser spots. Interestingly, one sees that most of the spots to the SW are red-shifted, while most of those to the NE are blue-shifted, consistent with the disk rotation velocity traced by methyl cyanide. This result is seen also in the PV plot in Fig. 11, where the points representing the $\mathrm{H}_{2} \mathrm{O}$ maser spots are overlaid on the PV plot of the $\mathrm{CH}_{3} \mathrm{CN} K=3$ component and compared to the pattern of a Keplerian disk rotating about a $7 M_{\odot}$ star. One sees that 22 out of $26(85 \%)$ of the SW spots are red shifted, and 17 out of $25(68 \%)$ of the NE spots are blue shifted, and their velocities are consistent with those of the $\mathrm{CH}_{3} \mathrm{CN}$ emission. This is strong evidence that the jet is tightly connected to the disk, at least on scales as small as a few 100 AU.

In conclusion, we stress that our findings not only indicate that the jet in IRAS $20126+4104$ is rotating about its axis, but also confirm that the velocity gradient observed in $\mathrm{CH}_{3} \mathrm{CN}$ is due

\footnotetext{
5 Unlike C2013, we made no attempt to improve on the relative astrometrical accuracy between the $\mathrm{CH}_{3} \mathrm{CN}$ and $\mathrm{H}_{2} \mathrm{O}$ positions. However, we caution the reader that the relative positional error may be as large as a significant fraction of the PdBI beam $\left(\lesssim 00^{\prime} 3\right)$.
} 


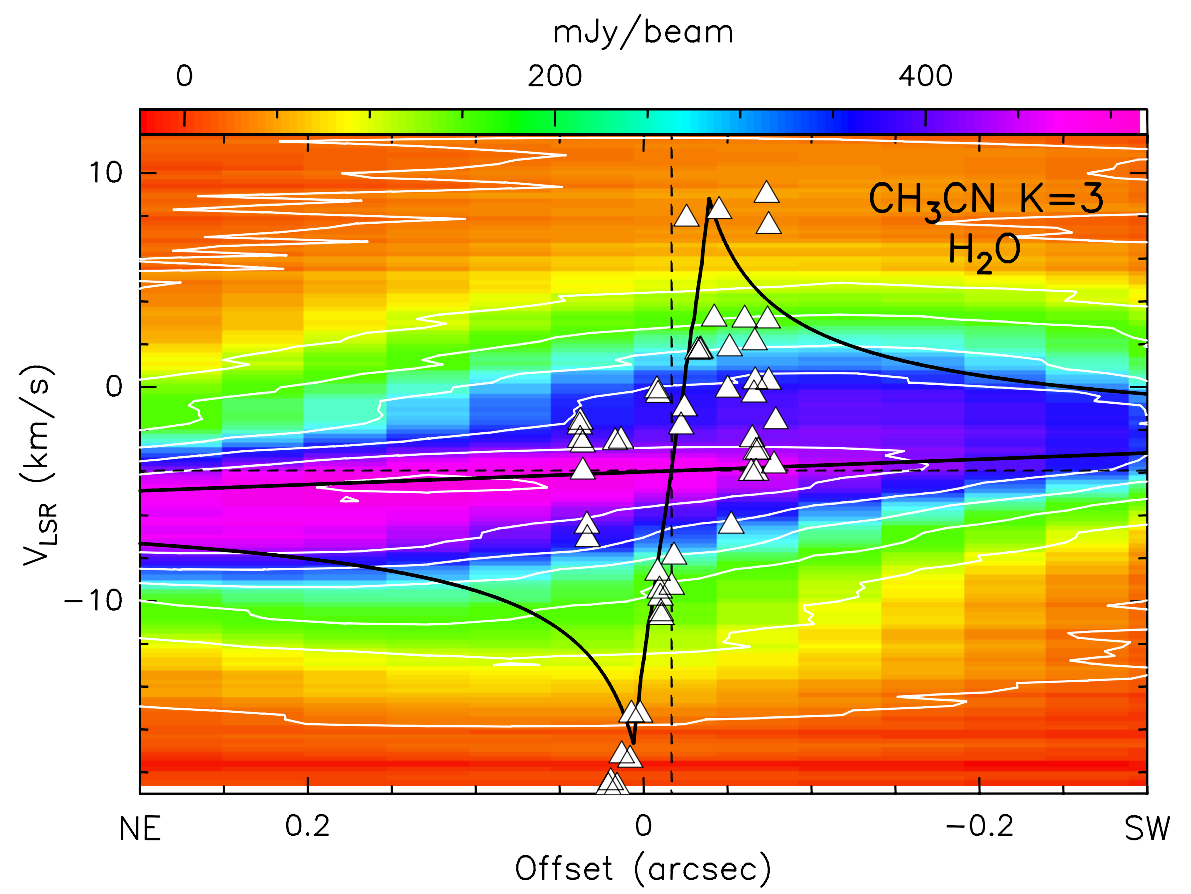

Fig. 11. Same as Fig. 6 for the $\mathrm{CH}_{3} \mathrm{CN} K=3$ line, with overlaid points (triangles) representing the $\mathrm{H}_{2} \mathrm{O}$ maser spots shown in Fig. 10. to rotation about the same axis. This result strongly indicates that the methyl cyanide emission is tracing a circumstellar rotating disk around IRAS 20126+4104.

\section{Summary and conclusions}

We performed interferometric observations of methyl cyanide rotational transitions towards the high-mass protostar IRAS 20126+4104, for which convincing evidence for the existence of a circumstellar disk had been found. Our results confirm that the $\mathrm{CH}_{3} \mathrm{CN}$ emission is indeed tracing a (quasi-)Keplerian disk rotating about a 7-10 $M_{\odot}$ protostar and demonstrate that the material is asymmetrically distributed in the plane of the disk.

We show that gravitational interactions with a nearby lowmass companion could significantly affect the disk structure and hence determine the observed disk asymmetry. Since the stellar cluster associated with IRAS 20126+4104 is known to be less populated than average (Qiu et al. 2008), our findings suggest that tidal interactions might be very effective in perturbing disks around massive stars and thus explain why disks around O-stars, surrounded by rich clusters, have been until now so elusive.

Finally, comparison between previous $\mathrm{H}_{2} \mathrm{O}$ maser data tracing the jet from IRAS 20126+4104 and our new $\mathrm{CH}_{3} \mathrm{CN}$ data reveal that the jet is rotating about its axis. This finding not only constrains the genesis of the jet, but also provides us with further, iron-clad evidence that the velocity gradient observed in $\mathrm{CH}_{3} \mathrm{CN}$ is indeed tracing rotation about the jet axis.

Acknowledgements. The authors gratefully acknowledge the IRAM technical staff for their support in this project. It is also a pleasure to thank Francesca Bacciotti for stimulating discussions about the physics of jets from YSOs.

\section{References}

Beltrán, M. T., Codella, C., Viti, S., Neri, R., \& Cesaroni, R. 2011, A\&A, 525, A151

Bruderer, S., van der Marel, N., van Dishoeck, E. F., \& van Kempen, T. A. 2014, A\&A, 562, A26

Caratti o Garatti, A., Froebrich, D., Eislöffel, J., Giannini, T., \& Nisini, B. 2008, A\&A, 485, 137
Cesaroni, R., Felli, M., Testi, L., Walmsley, C. M., \& Olmi, L. 1997, A\&A, 325, 725

Cesaroni, R., Felli, M., Jenness, T., et al. 1999, A\&A, 345, 949

Cesaroni, R., Neri, R., Olmi, L., et al. 2005, A\&A, 434, 1039 (C2005)

Cesaroni, R., Galli, D., Lodato, G., Walmsley, C. M., \& Zhang, Q. 2007, in Protostars and Planets V, eds. B. Reipurth, D. Jewitt, \& K. Keil (Tucson: Univ. of Arizona Press), 197

Cesaroni, R., Massi, F., Arcidiacono, C., et al. 2013, A\&A, 549, A146 (C2013)

Chiang, E. I., \& Goldreich, P. 1997, ApJ, 490, 368

Cossins, P., Lodato, G., \& Clarke, C. J. 2009, MNRAS, 393, 1157

Coffey, D., Bacciotti, F., Ray, T. P., Eislöffel, J., \& Woitas, J. 2007, ApJ, 663, 350

Edris, K. A., Fuller, G. A., Cohen, R. J., \& Etoka, S. 2005, A\&A, 434, 213

Hildebrand, R. H. 1983, QJRAS, 24, 267

Hofner, P., Cesaroni, R., Rodríguez, L. F., \& Martí, J. 1999, A\&A, 345, L43

Hofner, P., Cesaroni, R., Olmi, L., et al. 2007, A\&A, 465, 197

Hosokawa, T., \& Omukai, K. 2009, ApJ, 691, 823

Keto, E. H., \& Zhang, Q. 2010, MNRAS, 406, 102

Kratter, K. M., Matzner, C. D., Krumholz, M. R., \& Klein, R. I. 2010, ApJ, 708, 1585

Krumholz, M. R., Klein, R. I., McKee, C. F., Offner, S. S. R., \& Cunningham, A. J. 2009, Science, 323, 754

Kuiper, R., Klahr, H., Beuther, H., \& Henning, Th. 2010, ApJ, 722, 1556

Kuiper, R., Klahr, H., Beuther, H., \& Henning, Th. 2011, ApJ, 732, 20

Lebrón, M., Beuther, H., Schilke, P., \& Stanke, Th. 2006, A\&A, 448, 1037

Leurini, S., Codella, C., Zapata, L., et al. 2011, A\&A, 530, A12

Lissauer, J. J., \& Stevenson, D. J. 2007, in Protostars and Planets V, eds. B. Reipurth, D. Jewitt, \& K. Keil (Tucson: Univ. of Arizona Press), 591

Moscadelli, L., Cesaroni, R., \& Rioja, M. J. 2000, A\&A, 360, 663

Moscadelli, L., Cesaroni, R., \& Rioja, M. J. 2005, A\&A, 438, 889

Moscadelli, L., Cesaroni, R., Rioja, M. J., Dodson, R., \& Reid, M. J. 2011, A\&A, 526, A66

Palla, F., \& Stahler, S. W. 1993, ApJ, 418, 414

Piétu, V., Guilloteau, S., \& Dutrey, A. 2005, A\&A, 443, 945

Planck Collaboration XXI 2011, A\&A, 536, A21

Pringle, J. E. 1981, ARA\&A, 19, 137

Qiu, K., Zhang, Q., Megeath, S. T., et al. 2008, ApJ, 685, 1005

Reid, M. J., Schneps, M. H., Moran, J. M., et al. 1988, ApJ, 330, 809

Sánchez-Monge, Á., Cesaroni, R., Beltrán, M. T., et al. 2013, A\&A, 552, L10

Shepherd, D. S., Yu, K. C., Bally, J., \& Testi, L. 2000, ApJ, 535, 833

Sridharan, T. K., Williams, S. J., \& Fuller, G. A. 2005, ApJ, 631, L73

Tang, Y.-W., Guilloteau, S., Piétu, V., et al. 2012, A\&A, 547, A84

Tobin, J. J., Hartmann, L., Bergin, E., et al. 2012, ApJ, 748, 16

van der Marel, N., van Dishoeck, E. F., Bruderer, S., et al. 2013, Science, 340 1199

van der Marel, N., van Dishoeck, E. F., Bruderer, S., \& van Kempen, T. A. 2014, A\&A, 563, A113

Wilson, T. L., \& Rood, R. 1994, ARA\&A, 32, 191 